\title{
SUR LA SUBSTITUTION D'UNE VALEUR EXCEPTIONNELLE PAR UNE PROPRIÉTÉ LACUNAIRE.
}

\author{
Par \\ F. SUNYER I BALAGUER
}

à Barcelona.

A ma très chère mère.

\section{Introduction.}

C'est dans une Note de J. Hadamard $[5]^{1}$ qu'on trouve, pour la première fois, l'indication qu'il $\mathrm{y}$ a des relations entre les lacunes et les valeurs exceptionnelles. Plus tard, L. Féjer [4], M. Biernacki [2] ${ }^{2}$ et G. Pólya [11] se sont occupés de la même question; cependant G. Pólya en parle seulement à la fin de son mémoire et encore très sommairement, en employant un procédé qui ne lui permet pas d'obtenir des précisions.

L'objet du présent travail est la démonstration des résultats, beaucoup plus précis, que j'ai énoncés, sur le même thème. dans deux Notes [12]. D'ailleurs quelques uns des résultats de ce travail sont plus précis que ceux que j'ai donnés dans mes Notes citées et d'autres sont énoncés avec des notations différentes. ${ }^{3}$

Je signale que j'ai obtenu des résultats semblables à ceux des chapitres I et Il, pour les valeurs prises dans une bande horizontale par une fonction entière représentée par une série de Dirichlet lacunaire. Ces résultats je les démontrerai dans un autre recueil.

Maintenant je veux préciser ici les sens de deux notations que nous emploierons continuellement: L'expression $\varepsilon(r)$ représentera toujours une quantité positive qui tend vers zéro avec $1 / r$, mais il faut tenir compte que dans une démonstration, et aussi dans une même formule, elle pourra représenter des quantités différentes ayant

\footnotetext{
1 Les numéros figurant entre crochets renvoient à la bibliographie de la fin du mémoire.

2 Il m'a été tout-à-faìt impossible de consulter ce mémoire de Biernacki, je ne connais d'autres détails sur ce travail que ceux indiqués par Pólya.

Je signale que dans ces Notes se sont glissées plusieurs fautes d'impression.

2-632081 Acta mathematica. $87 \quad$ nars 1952.
} 
toujours cette propriété de devenir infiniment petites avec $1 / r$ Le sens de $\varepsilon(r)$ est donc presque le même que celui de $o(1)$, mais nous utilisons $\varepsilon(r)$ afin d'indiquer la variable et la limite vers laquelle elle tend.

D'autre part la suite $\left\{l_{k}\right\}$ aura toujours les propriétés suivantes: les $l_{k}$ sont des entiers tels que $0=l_{0}<l_{1}<\cdots<l_{k}<\cdots$ et que $\lim l_{k}=\infty$.

Je ne peux pas terminer cette introduction sans accomplir l'agréable devoir d'exprimer ma sincère reconnaissance aux Prof. J. Hadamard et S. Mandelbrojt pour l'amabilité qu'ils ont démontrée envers moi et pour m'avoir donné leur précieuse opinion sur ce travail.

\section{Chapitre I.}

\section{Fonctions entières d'ordre fini.}

1. Soit $\varrho(r)$ une fonction telle que

$$
\lim _{r=\infty} \varrho(r)=\varrho_{0}, \quad \lim _{r=\infty}\left(r \varrho^{\prime}(r) \log r\right)=0,
$$

et soit aussi $f(z)$ une fonction entière telle que

$$
\varlimsup_{r=\infty} \frac{\log M(r, f)}{U(r)}=b \quad\left(U(r)=r^{(r)}\right),
$$

où $M(r, f)$ représente le maximum de $|f(z)|$ sur la circonférence $|z|=r$, alors, si $b=1$, nous dirons que $f(z)$ est d'ordre $\varrho_{0}$ et d'ordre précisé $\varrho(r)$ tandis que si $b=0$, nous dirons que $f(z)$ est d'ordre précisé inférieur à $\varrho(r)$.

Si comme d'habitude nous considérons que deux couples de fonctions $f_{0}(z), f_{1}(z)$ et $g_{0}(z), g_{1}(z)$ sont le même couple lorsque $f_{1}(z) / f_{0}(z) \equiv g_{1}(z) / g_{0}(z)$, on sait que si $F^{\prime}(z)$ est une fonction entière d'ordre entier $\varrho_{0}$ et d'ordre précisé $\varrho(r)$, quelles que soient les fonctions $f_{0}(z) \neq \equiv$ et $f_{1}(z)$ entières et d'ordre précisé inférieur à $\varrho(r)$ (sauf au plus pour un couple exceptionnel), on a

$$
\varlimsup_{r=\infty} \frac{n\left(r, 1 /\left(f_{0} F-f_{1}\right)\right)}{U(r)}>0,
$$

où, suivant Nevanlinna, $n(r, \mathrm{I} / f)$ représente le nombre de zéros de $f(z)$ dans le cercle $|z| \leq r$.

Avec des conditions lacunaires sur la série de Taylor qui représente $F(z)$ on peut assurer la non existence du couple exceptionnel. Dans cet ordre d'idées nous démontrerons, en premier lieu, le résultat suivant. 
Sur la substitution d'une valeur exceptionnelle par une propriété lacunaire.

Théorème I. Soit

$$
F(z)=\sum_{k=0}^{\infty} a_{k} z^{l_{k}}
$$

une fonction entière d'ordre entier $\varrho_{0}$ et d'ordre précisé $\varrho(r)$, et soit $D$ la densité maximum de la suite $\left\{l_{k}\right\}$. Si $\varrho_{0} D<1$, alors, quelles que soient les fonctions $f_{0}(z) \neq 0$ et $f_{1}(z)$ entières et d'ordre précisé inférieur à $\varrho(r)$, on a

$$
\varlimsup_{r=\infty} \frac{n\left(r, 1 /\left(f_{0} F-f_{1}\right)\right)}{U(r)} \geq B_{1} C\left(\varrho_{0}\right),
$$

où $B_{1}$ dépend uniquement de $\varrho_{0} D$ et où $C\left(\varrho_{0}\right)$ est la quantité définie dans [13, pag. 479].

Remarque. On ne peut pas affaiblir la condition $\varrho_{0} D<1$, car la fonction $\exp \left(z^{0^{0}}\right)$ vérifie $\varrho_{0} D=1$ et présente le couple exceptionnel $f_{0}(z) \equiv 1, f_{1}(z) \equiv 0$.

Démonstration. Fin appliquant un résultat de Bernstein [1, lemme II, pag. 179] on peut démontrer facilement l'existence d'une suite $\left\{R_{n}\right\}\left(\lim R_{n}=\infty\right)$ telle que

$$
\lim _{n=\infty} \frac{\log M\left(R_{n}, F\right)}{U\left(R_{n}\right)}=\lim _{n=\infty} \frac{\log M\left(R_{n}, H\right)}{U\left(R_{n}\right)}=1
$$

où, pour simplifier, $H(z) \equiv f_{0}(z) F(z)-f_{1}(z)$.

Soit $B$ une quantité qui vérifie

$$
\varlimsup_{r=\infty} \frac{n(r, 1 / H)}{U(r)}<B C\left(\varrho_{0}\right)
$$

nous supposerons que $B$ peut être choisi de telle manière qu'il vérifie aussi $B<1$, car si cela n'était pas possible l'affirmation du théorème serait satisfaite, puisque on verra que $B_{1} \leq 1$. En appliquant donc une précision que j'ai obtenue [13, lemme 1] d'un résultat de Wiman et en posant (comme toujours dans ce mémoire) $z=r e^{i x}$ on déduit, pour $\Omega^{-1} R_{n}<r<\Omega R_{n}$,

(2) $\quad \log |H(z)|<\left[\left(\frac{r}{R_{n}}\right)^{\varrho_{0}} \psi_{2}\left(R_{n}, H\right) \cos \left(\varrho_{0} \alpha-\varrho_{0} \alpha\left(R_{n}, H\right)\right)+\right.$

$$
\left.+B \Omega^{e_{0}+1}+\varepsilon\left(R_{n}\right)\right] U\left(R_{n}\right)
$$

D'ailleurs, utilisant de nouveau le lemme de Bernstein [1, lemme II] appliqué à $f_{0}(z)$, on peut obtenir les inégalités 
(3) $\quad \log |F(z)| \leq \stackrel{+}{\log }|H(z)|+\stackrel{+}{\log }\left|f_{1}(z)\right|+\log 2-\log \left|f_{0}(z)\right|<$

$$
\begin{aligned}
<\left[\left(\frac{r}{R_{n}}\right)^{\varrho_{0}} \psi_{2}\left(R_{n}, H\right) \cos \left(\varrho_{0} \alpha-\varrho_{0} \alpha\left(R_{n}, H\right)\right)+B \Omega^{e_{0}+1}+\varepsilon\left(R_{n}\right)\right]^{+} \\
\cdot U\left(R_{n}\right)+\varepsilon\left(R_{n}\right) U\left(R_{n}\right),
\end{aligned}
$$

valables, comme (2), pour $\Omega^{-1} R_{n}<r<\Omega R_{n}$, et où $\log ^{+} X=(\log X+|\log X|) / 2$ et de même $[A]^{+}=(A+|A|) / 2$.

Or, d'après une précision d'un résultat de Pólya [14, lemme 1,3], dans le domaine

$$
\begin{gathered}
\frac{R_{n}}{1+\varepsilon\left(R_{n}\right)}<r<\left(1+\varepsilon\left(R_{n}\right)\right) R_{n}, \\
\left|\alpha-\alpha\left(R_{n}, H\right)-\frac{\pi}{\varrho_{0}}\right|<\pi D+\varepsilon\left(R_{n}\right),
\end{gathered}
$$

il existe un point $z_{n}$ tel que

$$
\log \left|F\left(z_{n}\right)\right|>\left(1-\varepsilon\left(R_{n}\right)\right) U\left(R_{n}\right)
$$

Des inégalités (3) et (4) et d'après les propriétés de $\psi_{2}(r, H)[13$, lemme 1] on déduit, en divisant par $U\left(R_{n}\right)$ et en passant à la limite,

$$
1 \leq\left[\beta(B) \cos \left(\pi-\pi \varrho_{0} D\right)\right]^{+}+B
$$

Maintenant soit $B_{1}$ la quantité qui vérifie

$$
1=\left[\beta\left(B_{1}\right) \cos \left(\pi-\pi \varrho_{0} D\right)\right]^{+}+B_{1}
$$

de (5) on déduit que toute valeur de $B$ qui vérifie (1) vérifie aussi $B \geq B_{1}$ et, par suite, l'affirmation du théorème est démontrée.

2. Nous définirons la fonction $A\left(\varrho_{0}\right)$ comme la borne supérieure des quantités $A \leq \varrho_{0}$ qui satisfont à

$$
\max _{\pi / 2<x<\pi A}\left(\log [\cos (x+\pi)]+e A\left(7+3 \log \frac{\varrho_{0}}{e A}\right)+\left(\pi^{2} A^{2}-x^{2}\right)^{1 / 2}\right) \leq 0,
$$

on voit facilement que $1>A\left(\varrho_{0}\right)>1 / 2$ et que $\lim _{\varrho_{0}=\infty} A\left(\varrho_{0}\right)=1 / 2$.

Avec cette définition on peut démontrer un résultat, semblable au théorème 1 , dans lequel on exprime la condition lacunaire au moyen de la densité moyenne supérieure $\bar{D}^{*}$ de la suite $\left\{l_{k}\right\}[9$, pag. 352], au lieu d'utiliser la densité maximum $D$, à savoir 
Sur la substitution d'une valeur exceptionnelle par une propriété lacunaire.

Théorème II. Soit

$$
F(z)=\sum_{k=0}^{\infty} a_{k} z^{l_{k}}
$$

une fonction entière d'ordre entier $\varrho_{0}$ et d'ordre précisé $\varrho(r)$. Si $\varrho_{0} \bar{D}^{*}<A\left(\varrho_{0}\right)$, quelles que soient les fonctions $f_{0}(z) \neq 0$ et $f_{1}(z)$ entières et d'ordre précisé inférieur à $\varrho(r)$, on a

$$
\varlimsup_{r=\infty} \frac{n\left(r, 1 /\left(f_{0} F-f_{1}\right)\right)}{U(r)} \geq B_{2} C\left(\varrho_{0}\right),
$$

où $B_{2}$ dépend uniquement de $\varrho_{0}$ et de $\bar{D}^{*}$.

Démonstration. On peut répéter ici les raisonnements de la démonstration du théorème I jusqu'à la formule (3) et on peut continuer de la façon suivante:

Selon Mandelbrojt [9, théorème $a$, pag. 366], quel que soit le point $z_{1}$, à l'intérieur du domaine défini par $\left|\log z-\log z_{1}\right| \leq \pi \bar{D}^{*}+\varepsilon$, où $\varepsilon$ est une quantité positive arbitraire, il existe un point $z^{\prime}$ pour lequel on a

$$
\log \left|F\left(z^{\prime}\right)\right|>\log \left|a_{k}\right|+l_{k} \log r_{1}-\log A_{k}^{*}-\log \left[\left(\pi \bar{D}^{*}+\varepsilon\right) L\left(\pi \bar{D}^{*}+\varepsilon\right)\right]^{1},
$$
où $r_{1}=\left|z_{1}\right|$.

D'ailleurs, à partir d'une valeur de $k$, d'après un résultat de Mandelbrojt [9, pag. 355] et puisque, d'après Dvoretzky [3], $D^{*} \leq e \bar{D}^{*}$, on a

$$
\frac{-\log \Lambda_{k}^{*}-\log \left[\left(\pi \bar{D}^{*}+\varepsilon\right) L\left(\pi \bar{D}^{*}+\varepsilon\right)\right]}{l_{k}}>-e \bar{D}^{*}\left(7-3 \log \left(e \bar{D}^{*}\right)\right)-\varepsilon .
$$

De plus, si l'on écrit $\mu(r)=\max _{0 \leq k<\infty}\left|a_{k}\right| r l_{k}$, on voit que, pour $r_{1}$ suffisamment grand, de $(6)$ et (7) il résulte que

$$
\log \left|F\left(z^{\prime}\right)\right|>\log \mu\left(r_{1} e^{h-\varepsilon}\right),
$$

où $h=-e \bar{D}^{*}\left(7-3 \log \left(e \bar{D}^{*}\right)\right)$. Or Valiron [15, pag. 32] démontre que pour les fonctions entières d'ordre fini on a $\log \mu(r)=(1-\varepsilon(r)) \log M(r, F)$ et, par suite, nous pourrons écrire

$$
\log \left|F\left(z^{\prime}\right)\right|>\left(1-\varepsilon\left(r_{1}\right)\right) \log M\left(r_{1} e^{h-\varepsilon}, F\right)
$$

\footnotetext{
1 Pour simplifier, nous posons $L(t)$ au lieu de $L_{n}(t)$ comme l'écrit Mandelbrojt, ce qui est permis, car, en définissant $L(t)$ à partir de $\Lambda(r)$ de la même façon que Mandelbrojt défini $L_{n}(t)$ à partir de $A_{n}(r)$, on voit facilement que $L(t)>L_{n}(t)$. Nous définirons la fonction $A(r)$ à partir de la suite $\left\{l_{k}\right\}$ presque de la même manière que Mandelbrojt la définit à partir de la suite $\left\{\lambda_{n}\right\}$; en réalité l'existence de $l_{0}=0$ impose une petite modification dans la définition de $\Lambda(r)$ et, par suite, de $L(t)$ et $\Lambda_{k}^{*}$.
} 
Si nous prenons, dans $(3), \log \Omega=\pi \bar{D}^{*}-h+2 \varepsilon$, et, dans (8),

$$
z_{1}=R_{n} \exp \left(-h+\varepsilon+i\left(\alpha\left(R_{n}, H\right)+\frac{\pi}{\varrho_{0}}\right)\right),
$$

de (3) et (8), après passage à la limite et en tenant compte du fait que $\varepsilon$ est arbítraire, on déduit que toute $B$ qui satisfait à (1) satisfait aussi à

$$
1 \leq \beta(B) q+\Omega_{0}^{p_{0}+1} B,
$$

où $\log \Omega_{0}=\pi \bar{D}^{*}-h$ et où, si $\varrho_{0} \bar{D}^{*} \geq 1 / 2$,

$$
\log q=\max _{\pi \varrho_{0} \bar{D}^{*} \geq \varrho_{0} \alpha \geq \pi / 2}\left[\log \left(\cos \left(\varrho_{0} \alpha+\pi\right)\right)-\varrho_{0} h+\left(\left(\pi \varrho_{0} \bar{D}^{*}\right)^{2}-\varrho_{0}^{2} \alpha^{2}\right)^{1 / 2}\right],
$$

et, si $\varrho_{0} \bar{D}^{*}<1 / 2, q=0$. Puisque par hypothèse $\varrho_{0} \bar{D}^{*}<A\left(\varrho_{0}\right)$, dans tous les cas nous aurons $q<1$ et, en conséquence, si nous définissons $B_{2}$ par

$$
1=\beta\left(B_{2}\right) q+\Omega_{0}^{\mathrm{e}}+1 B_{2},
$$

on tire $B \geq B_{2}$, et puisque $q$ et $\Omega_{0}$ dépendent uniquement de $\bar{D}^{*}$ et de $\varrho_{0}$, il sera de même de $B_{2}$ et, par suite, le téorème est démontré.

Le théorème $I$ n'est pas compris dans le théorème II car il existe des suites $\left\{l_{k}\right\}$ pour lesquelles $1>\varrho_{0} D \geq \varrho_{\theta} \bar{D}^{*} \geq A\left(\varrho_{0}\right)$ et de même le théorème II n'est pas compris dans le théorème $I$ car pour d'autres suites $\varrho_{0} D \geq 1>A\left(\varrho_{0}\right)>\varrho_{0} \bar{D}^{*}$.

3. Lorsque la fonction $F^{\prime}(z)$ est à croissance très régulière par rapport à l'ordre précisé $\varrho(r)$ on peut, dans les deux théorèmes antérieurs, prendre, au lieu de la limite supérieure de $n(r, 1 / H) / U(r)$, la limite inférieure de cette même expression, mais, pour démontrer cette possibilité il faut obtenir préalablement une modification dans le même sens du lemme 1 de mon travail [13] que nous avons utilisé dans la démonstration des théorèmes antérieurs; voici l'énoncé du résultat requis.

Lemme a. Soit $f(z)$ une fonction entière d'ordre entier $\varrho_{0}$ et d'ordre précisé $\varrho(r)$. Si

et si

$$
\varliminf_{r=\infty} \frac{\log M(r, f)}{U(r)}=b>0
$$

$$
\varliminf_{r=\infty} \frac{n(r, 1 / f)}{U(r)}<\left(B C_{1}\left(\varrho_{0}\right)\right)^{3 e_{0}}
$$

où $C_{1}\left(\varrho_{0}\right)$ est une constante semblable à $C\left(\varrho_{0}\right)$, alors il existe une suite $\left\{R_{n}\right\}\left(\lim R_{n}=\infty\right)$ telle que, pour $\Omega^{-1} R_{n}<r<\Omega R_{n}$, 
Sur la substitution d'une valeur exceptionnelle par une propriété lacunaire.

$\log |f(z)|<\left[\left(\frac{r}{R_{n}}\right)^{\varrho_{0}} \psi_{3}\left(R_{n}, f\right) \cos \left(\varrho_{0} a-\varrho_{0} \alpha\left(R_{n}, f\right)\right)+\frac{\Omega^{e_{0}+1} B}{b}+\varepsilon\left(R_{n}\right)\right] \log M\left(R_{n}, f\right)$

avec

$$
\left(1-\varepsilon\left(R_{n}\right)\right)\left(1-\frac{B}{b}\right)<\psi_{3}\left(R_{n}, f\right) \dot{<}\left(1+\varepsilon\left(R_{n}\right)\right) \beta\left(\frac{B}{b}\right)
$$

où $\beta(X)$ est la même fonction que dans [13, lemme 1].

La démonstration de ce lemme est semblable à celle de [13, lemme 1].

L'énoncé des résultats correspondants aux théorèmes I et II dans le cas des fonctions à croissance très régulière est le suivant.

Théorème III. Soit la fonction

$$
F(z)=\sum_{k=0}^{\infty} a_{k} z^{l_{k}}
$$

entière d'ordre entier $\varrho_{0}$, d'ordre précisé $\varrho(r)$ et telle que

$$
\lim _{r=\infty} \frac{\log M(r, F)}{U(r)}=b>0 .
$$

Si l'une au moins des deux conditions: $1^{\circ}: \varrho_{0} D<1,2^{\circ}: \varrho_{0} \bar{D}^{*}<A\left(\varrho_{0}\right)$ est satisfaite, alors, quelles que soient les deux fonctions $f_{0}(z) \not 0$ et $f_{1}(z)$ entières et d'ordre précisé inférieur à $\varrho(r)$, on a

$$
\lim _{r=\infty} \frac{n\left(r, 1 /\left(f_{0} F-f_{1}\right)\right)}{U(r)} \geq\left(B_{3} C_{1}\left(\varrho_{0}\right)\right)^{3 e_{0}}
$$

où $B_{3}=b B_{1}$ quand c'est la condition $1^{\circ}$ qui cst satisfaite et $B_{3}=b B_{2}$ quand c'est la condition $2^{\circ}$ qui est satisfaite.

Remarque. De même que le théorème I, le théorème III dans le cas de la condition $1^{\circ}$ ne peut pas, à un certain point de vue, être amélioré, c'est-à-dire, la condition $\varrho_{0} D<1$ ne peut pas s'affaiblir.

La démonstration du théorème III est toute pareille à la démonstration des théorèmes I et II en utilisant le lemme a au lieu du lemme 1 de [13].

4. Nous donnerons une seule application de ces théorèmes et tout particulièrement du théorème III.

Lindelöf [8] démontre que la fonction

$$
F(z)=\sum_{n=2}^{\infty}\left[\frac{z}{n^{2} \log n^{2}}\right]^{n^{2}} \text { satisfait à } \lim _{r=\infty} \frac{\log M(r, F)}{r /(e \log r)}=1
$$


et signale qu'il serait intéressant de démontrer que cette fonction est de genre 1. Cela découle inmédiatement du théorème III puisque pour cette fonction $\bar{D}^{*}=0$ et avec cette condition le théorème nous permet d'affirmer

$$
\lim _{r=\infty} \frac{n(r, 1 / F)}{r /(e \log r)} \geq\left(B_{2} C_{1}\left(\varrho_{0}\right)\right)^{3 e_{0}}
$$

ce qui démontre que la fonction est de genre 1 comme suppose Lindelöf.

\section{Chapitre II.}

\section{Fonctions entières d'ordre infini.}

5. Les théorèmes $d u$ chapitre I peuvent s'étendre aux fonctions d'ordre infini. Pour cela j'utiliserai les ordres de K. L. Hiong [6]; à savoir: Si $f(z)$ est une fonction entière d'ordre infini et si $W(r)$ satisfait à

$$
\begin{gathered}
W\left(r+\frac{r}{\log W(r)}\right)<[W(r)]^{1+\varepsilon(r),} \\
\varlimsup_{r=\infty} \frac{\log _{2} M(r, f)}{\log W(r)}=1 \quad\left(\log _{2} X=\log \log X\right),
\end{gathered}
$$

nous dirons que $f(z)$ est d'ordre $W(r)$. Par similitude avec l'ordre fini, K. L. Hiong appelle ordre de $f(z)$ l'expression $(\log W(r)) / \log r$, pour diverses raisons nous ne suivrons pas cette terminologie.

Maintenant soit $K(l)$ la plus grande valeur de $k$ qui satisfait à $l_{k} \leq l$ et posons $D(l)=K(l) / l^{\mathbf{1}}$, alors on peut énoncer.

Théorème IV. Soit

$$
F(z)=\sum_{k=0}^{\infty} a_{k} z^{l_{k}}
$$

une fonction entière d'ordre infini et d'ordre $W(r)$. Si

$$
\varlimsup_{l=\infty} D(l) l^{\beta}<\infty \quad(0<\beta<1)
$$

et si $W_{0}(r), W_{1}(r)$ et $W_{2}(r)$, ordres respectifs de $f_{0}(z), f_{1}(z)$ et $f_{2}(z)$, sont tels que

$$
\begin{array}{ll}
W_{0}(r)<[W(r)]^{\theta^{\prime}} & \left(\theta^{\prime}<\min \left(1, \frac{\beta}{1-\beta}\right)\right), \\
W_{i}(r)<[W(r)]^{\theta_{1}} & \left(\theta_{1}<1, i=1,2\right),
\end{array}
$$

${ }^{1} D(l)$ est donc la fonction de densité [9] de la suite que l'on obtient en suprimant $l_{0}$ de la suite $\left\{l_{k}\right\}$. 
Sur la substitution d'une valeur exceptionnelle par une propriété lacunaire.

il est impossible (quelles que soient $f_{0}(z) \neq 0, f_{1}(z)$ et $f_{2}(z)$ dont les ordres satisfont aux conditions antérieures) que l'identité

$$
f_{0}(z) F(z)-f_{1}(z)=f_{2}(z) e^{\varphi(z)}
$$

soit satisfaite pour aucune fonction entière $\varphi(z)$.

Démonstration. D'après le résultat déjà utilisé de Mandelbrojt [9, théorème $a]$, dans le domaine $\left|\log z-\log z_{1}\right|<t$, et quel que soit $z_{1}$, il existe un point $z^{\prime}$ pour lequel

$$
\log \left|F\left(z^{\prime}\right)\right|>\log \left|a_{k}\right|+l_{k} \log r_{1}-\log \Lambda_{k}^{*}-\log [t L(t)] .
$$

En écrivant $\mu(r)=\max \left(\left|a_{k}\right| r^{l_{k}}\right)$, on tire de (10)

$$
\log \left|F\left(z^{\prime}\right)\right|>\log \mu\left(r_{1}\right)-\log A_{k}^{*}-\log [t L(t)],
$$

mais ici $k$ n'est pas quelconque comme dans (10), ici $k$ est une fonction de $r_{1}$.

D'ailleurs, si $\left\{R_{n}\right\}\left(\lim R_{n}=\infty\right)$ est une suite telle que

$$
\log M\left(R_{n}, F\right)>\left[W\left(R_{n}\right)\right]^{1-\varepsilon\left(R_{n}\right)},
$$

on démontre facilement que

$$
\log \mu\left(R_{n}\right)>\left[W\left(R_{n}\right)\right]^{1-\varepsilon\left(R_{n}\right)} .
$$

D'autre part, en appliquant à $f_{0}(z)$ le lemme 4 de mon mémoire [13] et en prenant

$$
t=\left[W\left(R_{n}\right)\right]^{-\theta^{\prime \prime \prime}} \quad\left(\theta^{\prime}<\theta^{\prime \prime}<\theta^{\prime \prime \prime}<\min \left(1, \frac{\beta}{1-\beta}\right)\right),
$$

on tire de (11), en appliquant aussi les résultats de Mandelbrojt [9, pag. 355] et $\left[10\right.$, lemme VI], qu'il existe une suite $\left\{r_{n}\right\}$ telle que

$$
R_{n}<r_{n}<R_{n}+\frac{R_{n}}{\log W\left(R_{n}\right)}
$$

et que tout point de la circonférence $|z|=r_{n}$ est à une distance inférieure à $e r_{n} t$ d'un point $z^{\prime}$ qui satisfait à

$$
\begin{aligned}
\log \left|f_{0}\left(z^{\prime}\right) F\left(z^{\prime}\right)-f_{1}\left(z^{\prime}\right)\right|>\left[W\left(R_{n}\right)\right]^{1-\varepsilon\left(R_{n}\right)}- \\
-\left[W\left(R_{n}\right)\right]^{(1-\beta)\left(1+\varepsilon\left(R_{n}\right)\right)}-\left[W\left(R_{n}\right)\right]^{\theta^{\prime \prime \prime}(1-\beta) / \beta}-\left[W\left(R_{n}\right)\right]^{\theta_{2}}>\left[W\left[R_{n}\right)\right]^{1-\varepsilon\left(R_{n}\right)}
\end{aligned}
$$

où $1>\theta_{2}>\max \left(\theta_{1}, \theta^{\prime \prime}\right)$.

Supposons maintenant que le théorème ne soit pas vrai, c'est-à-dire, que pour un choix de $f_{0}(z), f_{1}(z)$ et $f_{2}(z)$ satisfaisant aux conditions du théorème il existe une 
fonction entière $\varphi(z)$ telle que l'identité (9) soit satisfaite. Alors, au moyen d'une nouvelle application du lemme 4 de [13], cette fois à $f_{2}(z)$, on démontre, en utilisant en plus une formule de Caratheodory (voir par ex. [7, pag. 299]) et une formule déjà classique, que

$$
\Re[\varphi(z)]<[W(r)]^{1+e(r)},\left|\varphi^{\prime}(z)\right|<[W(r)]^{1+e(r)},
$$

où $\mathfrak{R}[Z]$ est la partie reelle de $Z$.

D'autre part; tout point $z^{\prime}$ qui satisfait à (12) doit satisfaire aussi à

$$
\mathfrak{R}\left[\varphi\left(z^{\prime}\right)\right]>\left[W\left(R_{n}\right)\right]^{1-\varepsilon\left(R_{n}\right)}
$$

Par suite, en rappelant que tout point de la circonférence $|z|=r_{n}$ est à une distance inférieure à $e r_{n} t$ d'un point $z^{\prime}$ satisfaisant à (12), il résulte que pour tout point de $|z|=r_{n}$ on peut écrire

$$
\Re[\varphi(z)] \geq \Re\left[\varphi\left(z^{\prime}\right)\right]-\left|z-z^{\prime}\right|\left|\varphi^{\prime}\left(z_{0}\right)\right|>\left[W\left(R_{n}\right)\right]^{1-\varepsilon\left(R_{n}\right)}
$$

où $z_{0}$ est compris entre $z$ et $z^{\prime}$. Cela est impossible, car sur toute circonférence $|z|=R$ il doit exister des points où $\mathfrak{N}[\varphi(z)] \leq \Re[\varphi(0)]$. Cette absurdité démontre le théorème IV.

6. Corollaire. Avec les mêmes conditions que dans le théorème IV et si en plus l'ordre $W(r)$ est tel que $\log W(r)$ soit une fonction convexe de $\log r$, alors on a

$$
\varlimsup_{r=\infty} \frac{\log n\left(r, 1 /\left(f_{0} F-f_{1}\right)\right)}{\log W(r)}=1 .
$$

Démonstration. D'après Hiong [6, pag. 260-263], si le corollaire n'était pas vrai avec les zéros de $f_{0}(z) F(z)-f_{1}(z)$ on peut former un produit canonique $f_{2}(z)$ tel que $\log \left|f_{2}(z)\right|<[W(r)]^{\theta}$ où $0<1$; et par suite l'identité (9) serait satisfaite pour ce produit canonique $f_{2}(z)$ et pour une fonction $\varphi(z)$ entière déterminée, ce qui d'après le théorème IV n'est pas possible.

7. Lorsque la fonction est à croissance régulière par rapport à l'ordre $W(r)$; c'est-à-dire lorsque

on peut obtenir le

$$
\lim _{r=\infty} \frac{\log _{2} M(r, F)}{\log W(r)}=1
$$

Théorème V. Avec les mêmes conditions que dans le corollaire de no. antérieur et si en plus $F(z)$ est à croissance régulière par rapport à l'ordre $W(r)$, on a 
Sur la substitution d'une valeur exceptionnelle par une propriété lacunaire.

$$
\lim _{r=\infty} \frac{\log n\left(r, 1 /\left(f_{0} F-f_{1}\right)\right)}{\log W(r)}=1 .
$$

La démonstration est presque la même que celle du théorème IV mais en utilisant le théorème de Boutroux-Cartan (voir par ex. [16, pag. 10]).

\section{Chapitre III.}

\section{Fonctions holomorphes dans un cercle.}

8. Dans les théorèmes de Schottky, de Landau etc. l'hypothèse commune est l'existence de deux valeurs exceptionnelles; dans ce chapitre je démontre qu'on peut, dans la plupart de ces théorèmes, substituer à cette hypothèse une autre qui affirme seulement l'existence d'une seule valeur exceptionnelle, à condition qu'on suppose que la série de Taylor qui représente la fonction est suffisamment lacunaire. En premier lieu nous donnerons le résultat correspondant à une généralisation du théorème de Schottky.

Posons pour simplifier l'écriture $s(\beta)=(1-\beta)^{-1 / 2}-1$, on peut énoncer ainsi le

Théorème VI. Soit

$$
F(z)=\sum_{k=0}^{\infty} a_{k} z^{l_{k}}
$$

une fonction holomorphe dans le cercle $|z|<1$. Si les zéros et les $\dot{l}_{k}$ satisfont aux conditions

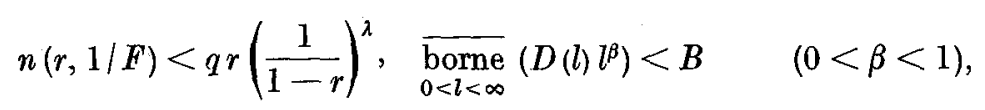

alors, quel que soit $\varrho>\max (\lambda, 1 / s(\beta))$, il existe une constante $r(q, \lambda, B, \beta, \varrho)<1$, qui sera définie dans la démonstration, telle que pour $r>r(q, \lambda, B, \beta, \varrho)$ l'inégalité

$$
\log M(r, F)<\left(\frac{e^{h}}{1-r}\right)^{e}+\log \frac{\left|a_{0}\right|}{e^{e}}
$$

soit satisfaite, où $h=3(\varrho+\varrho s(\beta)-1) /(\varrho s(\beta)-1)$.

Démonstration. La condition imposée aux zéros de $F(z)$ montre clairement que $\left|a_{0}\right| \neq 0$, par suite, nous pouvons raisonner sur la fonction $f(z)=e^{e} F(z) / a_{0}$ laquelle a les mêmes zéros et les mêmes $l_{k}$ que $F(z)$ et de plus satisfait à la condition $|f(0)|=e^{e}$. Comme il n'y a pas à craindre de confusions nous écrirons dans cette démonstration $M(r)$ et $n(x)$ au lieu respectivement de $M(r, f)$ et $n(r, 1 / f)$ et 
aussi $\mu(r)=\max _{0 \leq k<\infty}\left(e^{e}\left|a_{k}\right| r_{k} l_{k}|| a_{0} \mid\right)$. D'ailleurs nous définirons $r_{m}^{\prime}$ à partir de $r^{\prime}$ moyennant les égalités

$$
\frac{1}{1-r_{m}^{\prime}}=\frac{1}{1-r^{\prime}}\left(1+\frac{1}{\log _{2} M\left(r^{\prime}\right)}\right)^{m}
$$

Quel que soit $r$ il existe toujours, d'après un résultat de Borel, un $r^{\prime}$ tel que

$$
0<\log \frac{1}{1-r^{\prime}}-\log \frac{1}{1-r}<h,
$$

$$
\log M\left(r_{3}^{\prime}\right)<\left[\log M\left(r^{\prime}\right)\right]^{h /(h-3)}
$$

(on verra dans la suite pourquoi nous posons $r_{3}^{\prime}$ et $h /(h-3)$ au lieu de $r_{1}^{\prime}$ et $h /(h-1)$ ce quii paraîtrait plus naturel). Par suite, si $r$ satisfait à

$$
\log M(r)>\left(\frac{e^{h}}{1-r}\right)^{e}
$$

après des calculs faciles mais un peu longs, on arrive à

$$
M\left(r^{\prime}\right)<\left[\log M\left(r^{\prime}\right)\right]^{1 / e}\left[2\left(\log M\left(r^{\prime}\right)\right)^{h /(h-3)}+1\right]\left[\log _{2} M\left(r^{\prime}\right)+1\right] \mu\left(r^{\prime}\right) .
$$

De cette inégalité, si $1-r$, et par suite $1-r^{\prime}$, est plus petite qu'une constante positive qui dépend uniquement de $\varrho$ et $h$, et en appliquant de nouveau la première inégalité (13) et la (15), on tire

$$
\log \mu\left(r^{\prime}\right)>\frac{5}{6} \log M\left(r^{\prime}\right)
$$

Soit maintenant $\left\{x_{n}\right\}$ la suite des zéros de $f(z)$ à l'intérieur du cercle $|z|<1$ et soient aussi $P(z)$ et $\varphi(z)$ le polynome et la fonction holomorphe pour $|z|<r_{3}^{\prime}$ définis par

$$
P(z) \equiv \prod_{1}^{n}\left(1-\frac{z}{x_{n}}\right) \quad\left(n=n\left(r_{3}^{\prime}\right)\right), \quad e^{p(z)} \equiv \frac{f(z)}{P(z)}
$$

D'après le théorème de Boutroux-Cartan (voir par ex. [16, pag. 10]) l'on a $|P(z)|>$ $>(\eta / e)^{n}$, pourvu que l'on supprime du plan $n$ cercles dont la somme de diamètres est au plus $4 \eta$. De tout cela et en rappelant l'hypothèse du théorème sur $n(r)$ on démontre que si

$$
t=\left(\log M\left(r^{\prime}\right)\right)^{-\theta} \quad \text { avec } \quad \theta=\frac{\beta}{1-\beta} \frac{1}{\varrho(\varrho+1)},
$$

dans $|z| \leq r_{2}^{\prime}+t e$ on a $\mathfrak{R}[\varphi(z)]<2 \log M\left(r_{3}^{\prime}\right)$, pourvu que $1-r$ soit inférieur à une constante qui dépend uniquement de $h, \varrho, q, \lambda$ et $\theta$; et par suite, si cette constante 
Sur la substitution d'une valeur exceptionnelle par une propriété lacunaire.

est suffisamment petite, moyennant la formule de Caratheodory utilisée déjà dans le chapitre II, on peut démontrer que, dans $|z| \leq r^{\prime}+t e$,

$$
\left|\varphi^{\prime}(z)\right|<\left[\log M\left(r_{3}^{\prime}\right)\right]^{1+\frac{2}{\rho}+\theta}
$$

De (13), (14), (15) et (16) et des résultats de Mandelbrojt [9, théorème $a]$, [9, pag. $355]$ et [10, lemme VI] on tire que, si $1-r$ est inférieur à une constante qui dépend de $h, \varrho, 0, \beta$ et $B$, tout point de la circonférence $|z|=r^{\prime}$ est à une distance inférieure à $t e$ d'un point $z^{\prime}$ tel que

$$
\log \left|f\left(z^{\prime}\right)\right|>\frac{1}{2} \log M\left(r^{\prime}\right) .
$$

D'autre part, si $1-r$ est inférieur à une constante qui dépend de $\varrho, q$ et $\lambda$ on peut affirmer que $\log |P(z)|<\left(\log M\left(r^{\prime}\right)\right) / 6$ pour $|z| \leq r^{\prime}+t e$. Finalement, de (17) et (18) on tire donc que tout point de $|z|=r^{\prime}$ vérifie

$$
\Re[\varphi(z)]>\frac{1}{6} \log M\left(r^{\prime}\right)>e,
$$

pourvu que $1-r$ soit inférieur à une constante qui dépend uniquement de $h, q, \lambda$, $B, \beta$, $\varrho$ et 0 , c'est-à-dire, puisque $h$ et $\theta$ dépend uniquement de $\varrho$ et $\beta$, la (19) aura lieu pourvu que $r>r(q, \lambda, B, \beta, \varrho)$, ce qui est absurde puisque $\mathfrak{R}[\varphi(0)]=e$; cela démontre que pour ces valeurs de $r$ l'inégalité (15) ne peut pas être satisfaite. Le théorème est donc démontré.

9. Corollaire. Soit

$$
F(z)=\sum_{k=0}^{\infty} a_{k} z^{l_{k}}
$$

une fonction holomorphe dans $|z|<1$. Si

alors

$$
\varlimsup_{r=1} \frac{\log _{2} M(r, F)}{-\log (1-r)} \geq \varrho, \quad \overline{\underset{b<r n e}{\text { borne }}}\left(D(l) l^{\beta}\right)<\infty \quad\left(s(\beta) \geq \frac{1}{\varrho}\right),
$$

$$
\varlimsup_{r=1} \frac{\log n(r, 1 / F)}{-\log (1-r)} \geq \varrho .
$$

Ce corollaire est plutôt un énoncé moins précis du théorème VI.

10. Théorème VII. Soit une famille de fonstions holomorphes dans le cercle unité. Si toutes ces fonctions véritient les deux conditions

$$
n(r, 1 / f)<q r\left(\frac{1}{1-r}\right)^{\lambda}, \quad \overline{\text { borne }}\left(D(l) l^{\beta}\right)<\infty \quad(0<\beta<1),
$$


$q, \lambda, B$ et $\beta$ étant égales pour toutes les fonctions de la famille, celte famille est quasinormale à l'intérieur de $|z|<1$.

La démonstration est la classique à partir du théorème VI. Si en plus on suppose que les $|f(0)|$ sont également bornées, la famille est normale. Elle sera de même normale si l'on suppose que $n(r, 1 / f) \equiv 0$.

11. Théorème VIII. Soit une famille de fonctions $f(z)$ holomorphes dans le cercle unité et telle que chaque fonction possède une propriété $\Delta$ qui ne permet pas à la famille d'avoir des constantes parmi ses fonctions limites. Si, $\beta$ et $B$ étant égales pour toute la famille, la suite $\left\{l_{k}\right\}$ de chaque fonction est telle que

$$
\left.\underset{\substack{\text { borne } \\ 0<l<\infty}}{(D(l)} l^{\beta}\right)<B \quad(0<\beta<1) \text {, }
$$

il existe un cercle $d$ centré à l'origine, dont le rayon dépend uniquement de $\Delta$, de $\beta$ et de $B$, tel que toute fonction de la famille prend toute valeur $Y$ pourvu que $Y-f(0)$ soit intérieur au cercle $d$.

La démonstration résulte de la dernière affirmation du no. 10. Ce théorème, si $\Delta$ est la condition $f^{\prime}(0)=1$, diffère d'un théorème très connu de Koebe par le fait que la condition d'univalence est remplacée par une condition qui affirme que la série de Taylor est lacunaire.

12. Théorème IX. Soit

$$
F(z)=\sum_{k=0}^{\infty} a_{k} z^{l_{k}}
$$

une fonction holomorphe dans le cercle $|z|<R$. Si

$$
\left.n(r, 1 / F)<q\left(\frac{R}{R-r}\right)^{\lambda}, \quad \underset{\substack{\text { borne } \\ 0<l<\infty}}{(D}(l) l^{\beta}\right)<B \quad(0<\beta<1),
$$

$R$ doit être inférieur dane constante $R_{0}\left(a_{0}, a_{1}, l_{1}, q, \lambda, \beta\right)$.

La démonstration se fait comme d'habitude à partir du théorème VI.

\section{Bibliographie.}

1. Bernstein, V., Sulle direzioni di Borel di funzioni olomorfe, Annali di Mat. $4^{e}$ série, T. 12 (1934).

2. BiennackI, M., Sur les équations algébriques contenant des paramètres arbitraires, Thèse, Paris 1928.

3. Dvoretzky, A., Sur les suites d'exposants à densité supérieure finie, C. R. Acad. Sci. Paris, T. 225, pag. $481-483$ (1947). 
Sur la substitution d'une valeur exceptionnelle par une propriété lacunaire.

4. FÉJER, L., Über die Wurzel vom kleinsten absoluten Betrage einer algebraischen Gleichung, Mat. Annalen, T. 65, pag. 413-423 (1908).

5. Hadamard, J., Sur les fonctions entières, C. R. Acad. Sci. Paris, T. 136, pag. 1.309 -1.311 (1902).

6. Hiong, K. L., Sur les fonctions entières et les fonctions méromorphes d'ordre infini, Journ. de Math. $9^{\mathrm{e}}$ série, T. 14, pag. 233-308 (1935).

7. Landau, Handbuch der Lehre von der Verteilung der Primzahlen (Leipzig 1909).

8. Lindelöf, E., Mémoire sur la théorie des fonctions entières de genre fini, Acta Soc. Sci. Fenicae, T. 31 (1902).

9. Mandelbrojt, S.,. Sur une inégalité fondamentale, Ann. Sci. de l'Ec. Norm. Sup. $3^{\mathbf{e}}$ série, T. 63, pag. 351-378 (1947).

10. - Q Quasi-analyticity and analytic continuation - a general principle, Trans. of the Am. Math. Soc., Vol. 55. pag. 96-131 (1944).

11. Pólya, G., Untersuchungen über Lücken und Singularitäten von Potenzreihen, Math. Zeitschr., T. 29, pag. 549-640 (1929).

12. Sunyer I Balaguer, F., Sur la substitution d'une valeur exceptionnelle par une propriété lacunaire, C. R. Acad. Sci. Paris, T. 224, pag. 1609-1610 et T. 225, pag. $21-23(1947)$.

13. —- Sobre la substitucion de una funcion excepcional por una propiedad lagunar, Mem. de la R. Acad. de Cien. y Artes Barcelona, Tercera época, Vol. 29, pag. 475-516 (1948).

14. - Propiedades de las funciones enteras representadas por series de Taylor lagunares (Orden finite), Collectanea Math., Vol. 2, pag. 129-174 (1949).

15. VAlinon, G, Lectures of the general theory of integral functions, $2^{\mathrm{e}}$ édition, New York (1949).

16. —, Directions de Borel des fonctions méromorphes, Mém. Sci. Math. fasc. 89 (1938).

Séminaire Mathématique de l'Université de Barcelona, février 1951. 